\title{
Value of PTEN and Echocardiography in Predicting the Efficacy of Trimetazidine Combined with Metoprolol in the Treatment of Heart Failure
}

\author{
Xue-Hong Feng' \\ Yang Wang' \\ Lian-Yu Wang' \\ Jun-Fei Shen' \\ Chun-Yuan You' \\ Qiu-Ting Feng ${ }^{2}$
}

'Department of Echocardiography, The Second Hospital of Wuxi Affiliated to Nanjing Medical University, Wuxi, Jiangsu, 214002, People's Republic of China;

${ }^{2}$ Department of Cardiology, The Second Hospital of Wuxi Affiliated to Nanjing

Medical University, Wuxi, Jiangsu,

21 4002, People's Republic of China
Correspondence: Qiu-Ting Feng

Department of Cardiology, The Second Hospital of Wuxi Affiliated to Nanjing Medical University, No. 68 Zhongshan Road, Wuxi, 214002, Jiangsu Province,

People's Republic of China

Tel +86-15961823329

Email fengbiqu22538573@163.com
Objective: To investigate the predictive value of PTEN and echocardiography in the treatment of heart failure with trimetazidine combined with metoprolol.

Methods: A total of 100 patients with coronary heart disease and HF who admitted to our hospital from August 2018 to August 2020 were enrolled into research. All patients received routine treatment according to the guidelines and were treated with trimetazidine and metoprolol for a total course of 6 months. Echocardiographic parameters and PTEN levels were measured at baseline and after treatment. The patients were divided into groups according to the quartile of basic PTEN level, and the total effective rates were compared. The echocardiographic parameters of patients with different prognosis were analyzed. Bivariate correlation analysis was used to evaluate the correlation between PTEN, echocardiography and treatment effect.

Results: Compared with that before treatment, the level of PTEN increased significantly after treatment $(\mathrm{P}<0.01)$. According to the quartile of basic PTEN level, the total effective rate of patients with different levels of basic PTEN was was statistically different $(\mathrm{P}<0.01)$. There was a linear correlation between the level of basic PTEN and the treatment effect, and the total effective rate of patients with high level of basic PTEN was higher than that of patients with low level of PTEN. Compared with before treatment, LVEF, SV, E/A and lvfs increased significantly after treatment $(\mathrm{P}<0.01)$. There was a correlation between the basic echocardiographic parameters and the treatment effect of patients. The basic echocardiographic parameters of patients with poor prognosis were worse than those with good prognosis. PTEN expression in patients' serum was only positively correlated with E/A, but not with LVFE, SV and LVFS $(\mathrm{P}<0.01)$.

Conclusion: PTEN and echocardiographic parameters serve as a good method to evaluate the short-term therapeutic effect of trimetazidine combined with metoprolol in patients with heart failure.

Keywords: PTEN, echocardiographic parameter, heart failure, trimetazidine, metoprolol, efficacy prediction

\section{Introduction}

Heart failure (HF) is a familiar heart disease. It is very common in patients who die from HF all over the world. Recently, with aging world arriving, the incidence is also increasing. ${ }^{1,2}$ It's reported that the prevalence rate of HF is 3.1/1000; the morbidity has been increasing. ${ }^{3}$ HF can cause respiratory failure, hemoptysis, insomnia and nervous system diseases to some degree, and there are risks of sudden death in severe cases. ${ }^{4,5}$ Thus, HF diagnosis and treatment is crucial to the lives and health of patients. 
Myocardial fibrosis will cause imbalance of type I/III collagen, which will increase left ventricular stiffness, reduce compliance, and limit diastolic left ventricular filling; if the degree of myocardial fibrosis in patients continues to deepen, it will damage the systolic function of myocardium and greatly reduce the cardiac output. ${ }^{6,7}$ Echocardiography, as a non-invasive examination method, is easily accepted by patients; but it is difficult to make an accurate judgment on the distribution of myocardial fibrosis, because its detection results are easily influenced by subjective factors of operators. ${ }^{8}$ It has been found that echocardiography combined with biomarkers can improve the diagnosis rate of HF patients. ${ }^{9}$ PTEN (phosphatase and tensin homolog deleted on chromosome 10) is a tumor suppressor gene discovered early. ${ }^{10}$ Research has shown that PTEN regulates cell growth and apoptosis. ${ }^{11}$ For example, PTEN can adjust Akt activity by inhibiting PI3K, and play a regulatory role in apoptosis. ${ }^{12}$ PTEN modulation by hexarelin attenuates coronary artery ligation-induced HF in rats. ${ }^{13}$ However, whether PTEN has clinical value in HF has not been verified yet.

Trimetazidine combined with metoprolol is effective in the treatment of coronary heart disease and heart failure, serving as one of the commonly used clinical schemes. ${ }^{14}$ Here, in this study, we mainly explored the changes of PTEN and echocardiographic parameters after treatment in HF patients, and the potential predictive value of efficacy, aiming to provide reference indicators for clinical treatment and efficacy prediction.

\section{Methods and Data}

\section{Clinical Data}

100 patients with coronary heart disease and HF who admitted to our hospital from August 2018 to August 2020 were enrolled into research. This experiment has been ratified by the Ethics Committee of Second Hospital of Wuxi Affiliated to Nanjing Medical University, all methods were performed in accordance with the Declaration of Helsinki, and all the participants have signed informed consent forms.

\section{Inclusion and Exclusion Criteria}

Inclusion criteria: Over 45 years old; conformed to the clinical manifestations of chronic heart failure; diuretics, beta-receptor-blocking agent and spironolactone were given according to the 2014 Chinese guidelines for the diagnosis and treatment of heart failure; the cardiac function were grade III IV according to New York Heart Association (NYHA); echocardiography showed left ventricular ejection fraction (LVEF) $<50 \%$.

Exclusion criteria: Patients with other cardiovascular and cerebrovascular diseases, immune deficiency diseases, infectious diseases, mental disorders and tumor diseases; those with liver and kidney organ dysfunction; those with drug allergy; those who received surgery, radiotherapy and chemotherapy, and antibiotics within six months prior to admission; those transferred.

\section{Therapeutic Regimens}

After admission, all patients were given routine treatment intervention, including diuretics, nasal catheter oxygen inhalation, cardiotonic agents, correction of water and electrolyte disorders, and blood lipid lowering and vasodilatory drugs. On this basis, trimetazidine and metoprolol were used. The dosage of metoprolol tartrate tablets is $6.25 \mathrm{mg} / \mathrm{time}, 3$ times/d for the first time. After 14 days of treatment, the dosage should be appropriately increased or decreased according to patients' actual condition and recovery. It can be increased once every 7 days to 6.25 $12.5 \mathrm{mg}$. The defined daily dose should be controlled within $300 \mathrm{mg}$. In like wise, trimetazidine hydrochloride tablets should be $20 \mathrm{mg} /$ time, orally, 3 times/d, for a total of 6 months.

\section{Ultrasonic Testing}

The patient was examined by an experienced sonographer, with left lateral position. The left ventricular end systolic diameter (LVESD) and left ventricular end diastolic diameter (LVEDD) were obtained through the parasternal long axis section of the left ventricle with PHILIPS HD 11 echocardiography and C5-2 probe (frequency 2.0-5.0 $\mathrm{MHz}$ ). What's more, the left ventricular ejection fraction (LVEF) was calculated. The mitral E/A ratio was obtained by apical four-chamber section, and the results were averaged after 3 tests. The rate of left ventricular fractional shortening (LVFS) was tested by M-mode echocardiography.

\section{PTEN Expression}

QRT PCR was used. The serum to be tested was obtained; the total RNA was separated through TRIzol (Invitrogen, USA), and then $5 \mu \mathrm{L}$ was used to test its integrity under agarose gel electrophoresis. According to the reverse transcription kit instructions (BIOMIGA, USA), the total RNA was reverse transcribed into cDNA, and qRT-PCR was 
Table I Comparison of Basic Data of Patients with Different Prognosis

\begin{tabular}{|c|c|c|c|c|c|c|}
\hline & $\begin{array}{l}\text { Obvious Improvement } \\
\qquad(n=34)\end{array}$ & $\begin{array}{l}\text { General Improvement } \\
\qquad(n=38)\end{array}$ & $\begin{array}{l}\text { No Improvement } \\
(n=26)\end{array}$ & $\begin{array}{l}\text { Deterioration } \\
\qquad(n=2)\end{array}$ & $F / \chi^{2}$ & $P$ \\
\hline Age (year) & $62.94 \pm 3.56$ & $63.12 \pm 3.44$ & $62.87 \pm 3.62$ & $63.23 \pm 3.84$ & 8.932 & 0.864 \\
\hline $\begin{array}{l}\text { Gender/male } \\
{[n, \%]}\end{array}$ & 25 & 20 & 12 & I & 16.483 & 0.462 \\
\hline $\begin{array}{l}\text { Hypertension } \\
{[n, \%]}\end{array}$ & 23 & 21 & 10 & I & $|2.45|$ & 0.678 \\
\hline Diabetes[n, \%] & 23 & 19 & 14 & 2 & 10.678 & 0.563 \\
\hline
\end{tabular}

performed with GAPDH as internal reference (primer sequence, Invitrogen, USA) (Table 1). Reaction conditions: $95^{\circ} \mathrm{C}$ for $10 \mathrm{~min}$, followed by 40 cycles of $95^{\circ} \mathrm{C}$ for $15 \mathrm{~s}$ and $60^{\circ} \mathrm{C}$ for $1 \mathrm{~min}$. The reaction system: cDNA product $2 \mu \mathrm{L}, 50 \times \mathrm{ROX}$ reference dye $0.4 \mu \mathrm{L}$, SYBR qPCR Mix $10 \mu \mathrm{L}$, upstream and downstream primers 0.8 $\mu \mathrm{L}$ each, and RNase water made up to $20 \mu \mathrm{L}$. The circulation threshold (ct value) of the target gene and internal reference was read, and the relative level of the former in the sample was expressed by $2^{-\Delta \Delta \mathrm{Ct}} \cdot{ }^{15}$ PTEN upstream sequence 5'-CCGCTGTGTGTGGTGatatc-'3, downstream sequence 5'-GAATGTATTTACCCAAAAGTGAAATT-'3; GAPDH upstream sequence 5'-GGAAGGTGAGGTC GGAG-'3, downstream sequence 5'-CGTTCTCACCT GCGG-'3.

\section{Efficacy Evaluation}

According to the cardiac function at discharge, it can be divided into obvious improvement (cardiac function after treatment improves by more than 2 grades), general improvement (cardiac function after treatment improves by 1 grade), no improvement (no improvement of cardiac function after treatment) and deterioration (aggravation of heart failure, deterioration or death after treatment). Total effective $=$ obvious improvement + general improvement .

\section{Statistical Analysis}

All the data were statistically analyzed by SPSS22.0 statistical software. The relevant pictures were drawn by GraphPad 8 software. The average value was obtained after 3 repetitive tests, recorded as (mean \pm standard deviation). The comparison between groups was carried out by independentsamples $t$-test, including $\mathrm{t}$ value and $P$ value. According to the basic PTEN level grouping, the two-way ordered classification data linear trend test was used to compare the improvement of the overall clinical status of patients with different prognosis after treatment. Logistic regression model was used to evaluate the predictive value of PTEN and echocardiography on the efficacy of trimetazidine combined with metoprolol in the treatment of heart failure. $P$ value of $<0.050$ is considered as statistical difference.

\section{Results Comparison of Basic Data of Patients with Different Prognosis}

Among $100 \mathrm{HF}$ patients, there were 58 males and 42 females, aged from 48 to 68 years, with an average age of $(60.13 \pm 5.84)$ years, 55 cases of hypertension, and 58 cases of diabetes. After treatment of trimetazidine combined with metoprolol, there were 34, 38, 26, 2 cases of obvious improvement, general improvement, no improvement and deterioration respectively. There was no significant difference in the basic data of patients with different prognosis $(\mathrm{P}>0.05)$. Table 1

\section{Expression of PTEN in HF Patients}

Compared with before treatment, the level of PTEN increased significantly after treatment $(\mathrm{t}=24.284, \mathrm{P}<$ 0.01). The basic PTEN level of the included patients was $0.21 \sim 0.76[0.47(0.63)]$. According to the quartile of basic PTEN level, they were divided into $\leq 0.34$ group (23 cases), $0.35 \sim 0.53$ ( 29 cases), $0.54 \sim 0.64$ (30 cases) and $\geq 0.65$ (18 cases). The total effective rates of patients with different levels of basic PTEN were compared, and the difference was statistically significant $(\mathrm{P}<0.01)$. The linear trend test results of ordered grouping data showed that there was a linear correlation between the basic PTEN level and the treatment effect of patients $\left(\chi^{2}\right.$ total $=16.372$, $\mathrm{P}=0.031 ; \chi_{\text {Regression }}^{2}=9.684, \mathrm{P}=0.014 ; \chi^{2}$ bias $=5.382$, $\mathrm{P}=0.46 \sim 0.78)$. The total effective rate of patients with high level of basic PTEN was higher than that of patients with low level of PTEN. Figure 1, Table 2 


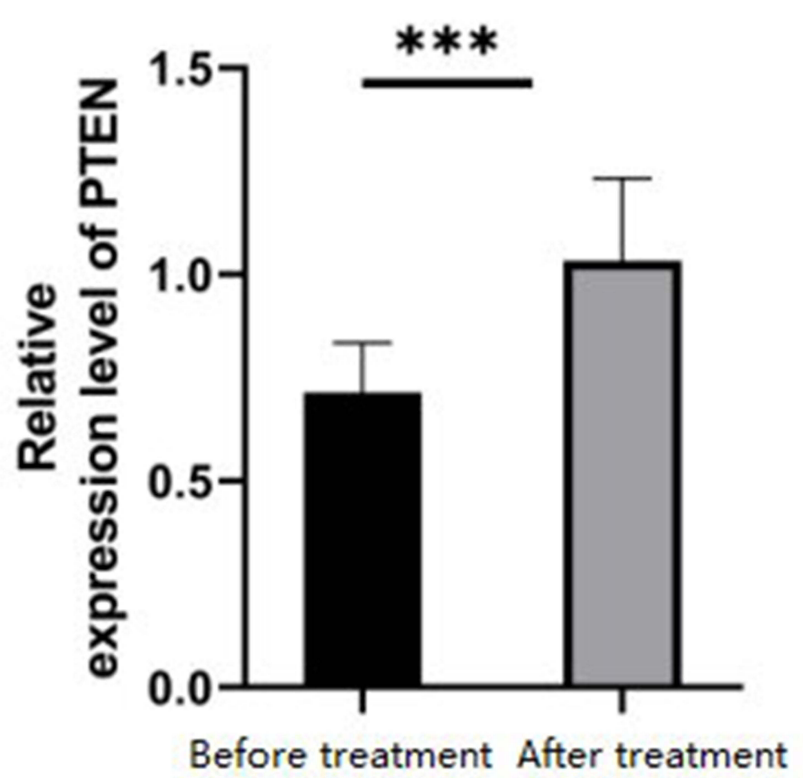

Figure I Expression of PTEN in HF patients. ***P<0.00I.

\section{Echocardiographic Parameters in HF Patients}

Compared with before treatment, LVEF, SV, E/ A and LVFS increased significantly after treatment $(\mathrm{t}=21.324,19.463$, 24.673, $\mathrm{P}<0.01)$. The linear trend test of ordered grouping data showed that there was a correlation between basic echocardiographic parameters and treatment effect $\left(\chi^{2}\right.$ total $=11.674, \mathrm{P}=0.035 ; \chi^{2}$ Regression $=14.275, \mathrm{P}=0.018 ; \chi^{2}$ bias $=8.928, \mathrm{P}=0.32 \sim 0.76)$. The basic echocardiographic parameters of patients with poor prognosis were worse than those with good prognosis. Figure 2, Table 3

\section{Correlation Between PTEN and \\ Echocardiographic Parameters}

At the end of the study, we analyzed the relationship between serum PTEN and echocardiographic parameters in HF patients. Through analysis, we found that PTEN expression in patients' serum was only positively correlated with E/A, but not with LVFE, SV and LVFS (Figure 3, $P<0.05$ ).

\section{Discussion}

Drug therapy is the main treatment for coronary heart disease and HF, but the efficacy of conventional drugs is not good. Further improving the science and rationality of medication is of great significance to improving the effectiveness of treatment. Metoprolol is a commonly used clinical $\beta 1$-receptor blocker and a first-line antihypertensive drug. Compared with other anti-angina pectoris drugs, trimetazidine has less effect on myocardial hemodynamics, and can significantly improve endothelial function in patients with heart failure and relieve clinical symptoms. Relevant studies have shown that metoprolol combined with trimetazidine has a significant effect in the treatment of patients with coronary heart disease and HF, and can effectively improve the hemodynamics of patients. ${ }^{16}$ In this study, we examined the predictive value of PTEN and echocardiographic parameters in HF patients after treatment. PTEN is a bifunctional phosphatase, which can dephosphorize lipid and protein. It negatively regulates PI3K pathway by dephosphorizing phosphatidylinositol triphosphate and pAkt, thus regulating apoptosis and cell proliferation pathway. ${ }^{17}$ Furthermore, recent research has demonstrated that PTEN down-regulation can participate in smooth muscle cell (SMC) proliferation and vascular remodeling, which is considered to be involved in the occurrence of cardiac fibrosis after myocardial infarction. ${ }^{18}$ Echocardiography is the most common clinical method for evaluating cardiac function, which is rapidly popularized because of its non-invasive, simple, repeatable and relatively low cost. ${ }^{19}$ The comprehensive parameters of echocardiography include many factors, such as cardiac systolic function, cardiac diastolic function, ventricular remodeling, valve regurgitation, pulmonary artery pressure, etc.; considering the mutual influence and action among various parts, the evaluation result is not

Table 2 Comparison of Curative Effects of Patients with Different Levels of Basic PTEN n/\%

\begin{tabular}{|l|c|c|c|c|c|c|}
\hline $\begin{array}{l}\text { PTEN } \\
\text { Grouping }\end{array}$ & $\mathbf{n}$ & $\begin{array}{c}\text { Obvious } \\
\text { Improvement }\end{array}$ & $\begin{array}{c}\text { General } \\
\text { Improvement }\end{array}$ & $\begin{array}{c}\text { No } \\
\text { Improvement }\end{array}$ & Deterioration & $\begin{array}{c}\text { Total } \\
\text { Effectiveness }\end{array}$ \\
\hline$\leq 0.34$ & 23 & 3 & 12 & 6 & 2 & $15 / 65.22$ \\
$0.35 \sim 0.53$ & 29 & 6 & 13 & 10 & 0 & $19 / 65.52$ \\
$0.54 \sim 0.64$ & 30 & 13 & 8 & 9 & 0 & $21 / 70.00$ \\
$\geq 0.65$ & 18 & 12 & 5 & 26 & 0 & $17 / 94.44$ \\
Count & 100 & 34 & 38 & 1 & 2 & $72 / 72.00$ \\
\hline
\end{tabular}



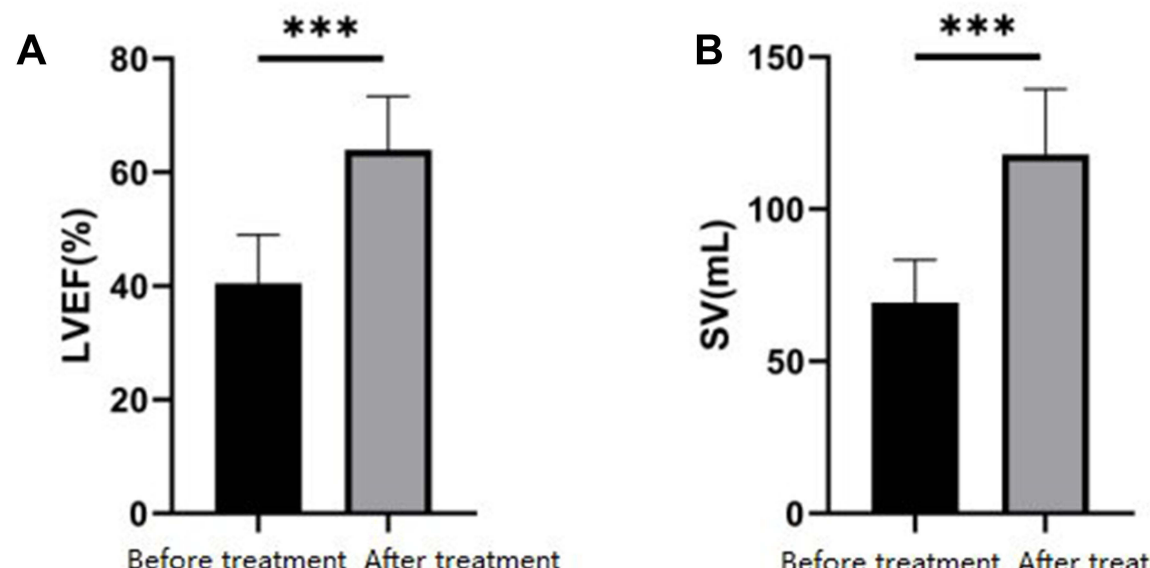

Before treatment After treatment
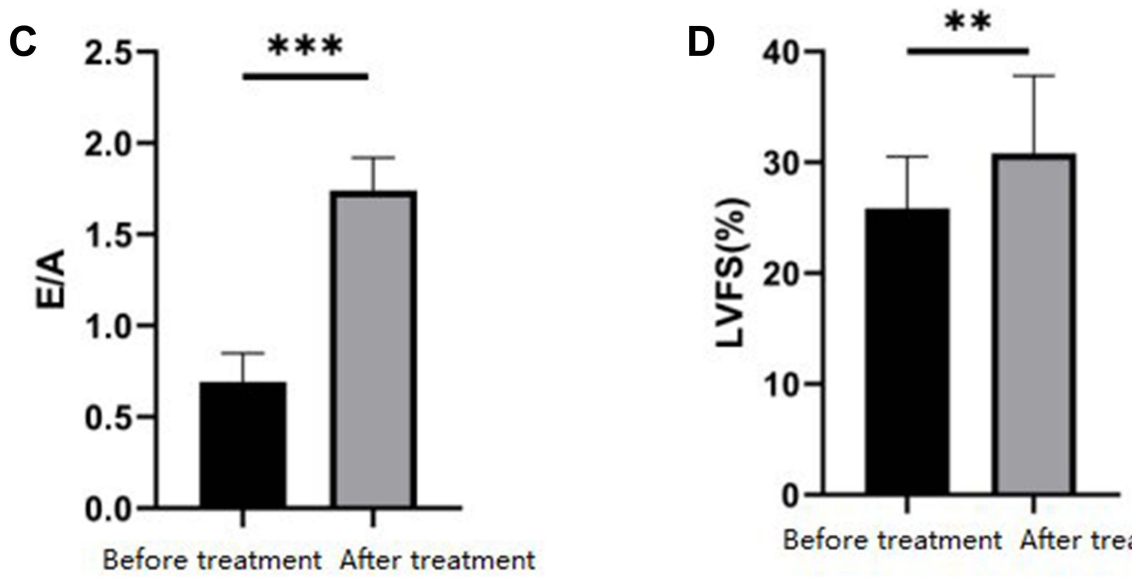

Before treatment After treatment

Figure 2 Echocardiographic parameters in HF patients. (A-D) Measurement of LVEF, SV, E/A and LVFS parameters in HF patients under echocardiography. **P $<0.01$, $* * * \mathrm{P}<0.001$.

a simple numerical accumulation, but an overall evaluation of cardiac function made by integrating the changes of various parts of the heart. ${ }^{20}$ We also found that compared with before treatment, PTEN levels increased significantly after treatment, suggesting that PTEN and echocardiographic parameters may be related to the occurrence of heart failure. And compared with before treatment, LVEF, $\mathrm{SV}, \mathrm{E} / \mathrm{A}$, and LVFS were significantly increased after treatment, which was in line with the results of previous studies, suggesting that metoprolol combined with trimetazidine may affect PTEN levels and improve patients' heart function. Early prognosis evaluation is a crucial means to improve the efficacy and prognosis of patients. Afterwards, patients choose a more favorable treatment plan, which can effectively control the disease, speed up the recovery, and avoid poor efficacy or prognosis caused by untimely treatment. ${ }^{21}$ Here, we divided the patients into groups according to their clinical efficacy after treatment.

Table 3 Comparison of Basic Echocardiographic Parameters in Patients with Different Prognosis

\begin{tabular}{|l|c|c|c|c|c|}
\hline Groups & $\mathbf{n}$ & LVEF/\% & SV/mL & E/A & LVFS/\% \\
\hline Obvious improvement & 34 & $45.39 \pm 3.87$ & $54.32 \pm 2.21$ & $0.48 \pm 0.05$ & $28.59 \pm 1.24$ \\
General improvement & 38 & $44.67 \pm 3.68$ & $52.73 \pm 4.14$ & $0.41 \pm 0.04$ & $27.57 \pm 1.18$ \\
No improvement & 26 & $40.27 \pm 2.56$ & $44.37 \pm 3.96$ & $0.26 \pm 0.02$ & $24.58 \pm 1.14$ \\
Deterioration & 2 & $39.41 \pm 2.33$ & $42.69 \pm 3.78$ & $0.24 \pm 0.03$ & $23.96 \pm 1.15$ \\
F & & & & & \\
$P$ & & & & & \\
\hline
\end{tabular}



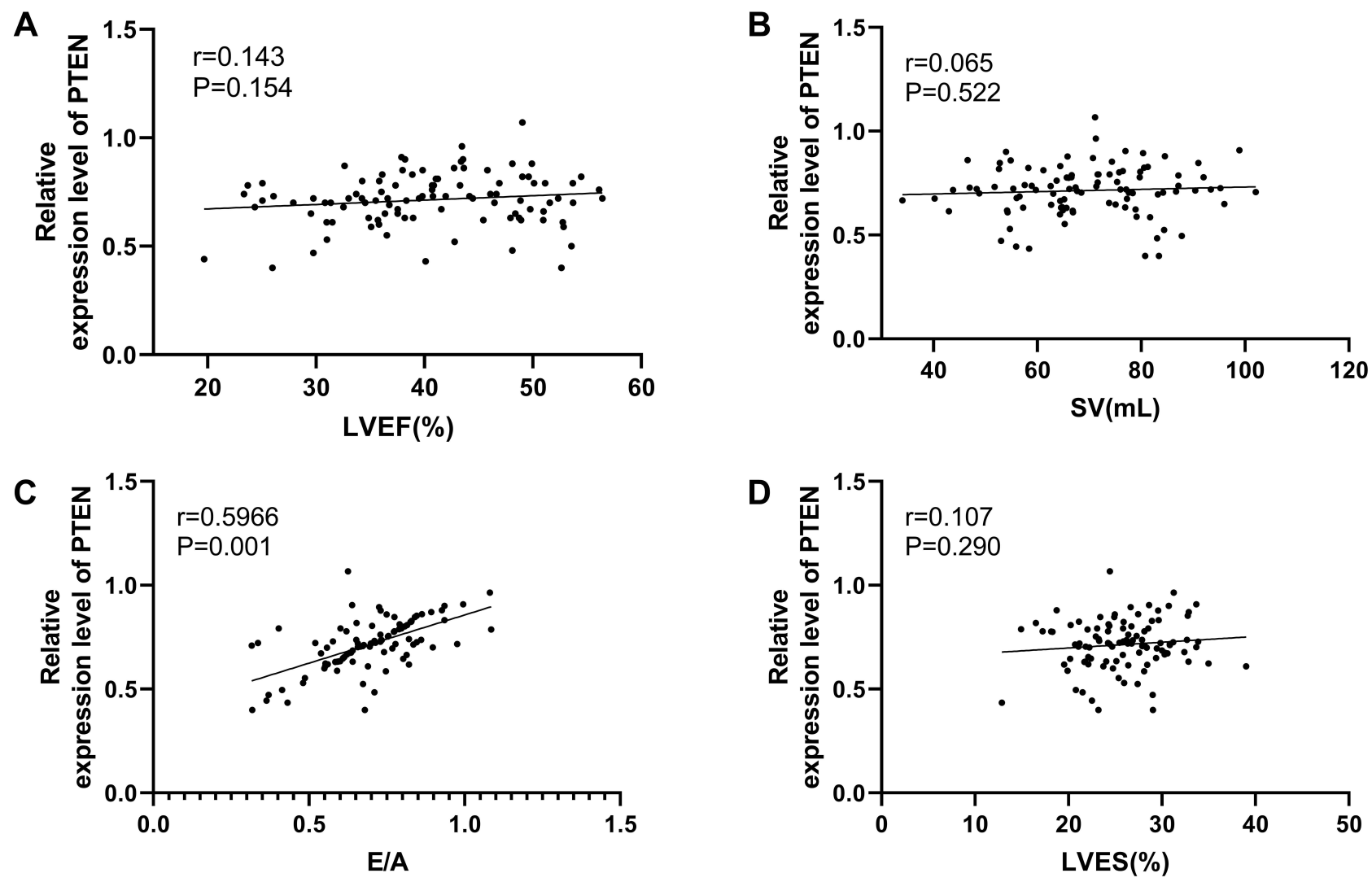

Figure 3 Correlation between PTEN and echocardiographic parameters. (A) Correlation analysis of serum PTEN relative level and LVEF in HF patients. (B) Correlation analysis of serum PTEN relative level and SV in HF patients. (C) Correlation analysis of serum PTEN relative level and E/A in HF patients. (D) Correlation analysis of serum PTEN relative level and LVES in HF patients.

Based on the quartile of basic PTEN level, we found that the total effective rate of patients with high basic PTEN level was higher than that of patients with low basic PTEN level, suggesting that patients with high basic PTEN level may have a better prognosis, which can provide a reference for the formulation of clinical treatment plan; the basic echocardiographic parameters of patients with poor prognosis were worse than those with good prognosis, indicating that echocardiographic parameters are expected to become a potential index to predict the clinical efficacy of patients. The main feature of ventricular remodeling after myocardial infarction is that in the initial stage, myocardial cell death and inflammation around and in the infarcted area increase, leading to myocardial hypertrophy and fibrosis, which is considered to be a key process involved in the pathophysiology of $\mathrm{HF}^{22,23}$ Early studies have suggested that PTEN deletion brings about myocardial hypertrophy. ${ }^{24}$ We believe that HF patients with low PTEN expression predict myocardial fibrosis and hypertrophy, which is not conducive to the improvement of patients' condition. The E/A ratio is one of the indexes for the auxiliary diagnosis of HF with normal left ventricular ejection fraction; ${ }^{25}$ have verified that the ratio is used to evaluate essential hypertension with HF and hypertension-induced left ventricular hypertrophy. Through correlation analysis, we also determined that there was a positive correlation between PTEN and E/A, which further indicates that PTEN is expected to become a predictor of efficacy in HF patients.

Besides, we confirmed the predictive value of PTEN and echocardiographic parameters in HF patients after treatment. In this study, we found that PTEN expression in patients' serum was only positively correlated with E/A, but not with LVFE, SV and LVFS. But, this study still has some limitations. First of all, this study did not conduct a retrospective study of large samples. Thus, we hope to verify the study through large sample data. Secondly, this study did not analyze the mechanism of PTEN in HF. We hope to carry out basic research in the follow-up study to determine the mechanism.

Above all, PTEN and echocardiographic parameters can better evaluate the short-term therapeutic effect of 
trimetazidine combined with metoprolol in patients with heart failure.

\section{Funding}

This study was supported by the Cultivation of Key Young Medical Talents in Jiangsu Province (Jiangsu Provincial Health and Family Planning Commission QNRC2016142).

\section{Disclosure}

The authors report no conflicts of interest in this work.

\section{References}

1. Bozkurt B, Khalaf S. Heart failure in women. Methodist DeBakey Cardiovasc J. 2017;13(4):216-223. doi:10.14797/mdcj-13-4-216

2. Arrigo M, Ruschitzka F, Flammer AJ. [Acute heart failure]. Ther Umsch. 2018;75(3):155-160. Deutsch. doi:10.1024/0040-5930/ a000980

3. Sinnenberg L, Givertz MM. Acute heart failure. Trends Cardiovasc Med. 2020;30(2):104-112. doi:10.1016/j.tcm.2019.03.007

4. Price JF. Congestive heart failure in children. Pediatr Rev. 2019;40 (2):60-70. doi:10.1542/pir.2016-0168

5. Tomasoni D, Adamo M, Lombardi CM, Metra M. Highlights in heart failure. ESC Heart Fail. 2019;6(6):1105-1127. doi:10.1002/ ehf2.12555

6. Yancy CW, Jessup M, Bozkurt B, et al. 2013 ACCF/AHA guideline for the management of heart failure: a report of the American college of cardiology foundation/American heart association task force on practice guidelines. J Am Coll Cardiol. 2013;62(16):e147-e239. doi:10.1016/j.jacc.2013.05.019

7. Snipelisky D, Chaudhry SP, Stewart GC. The many faces of heart failure. Card Electrophysiol Clin. 2019;11(1):11-20. doi:10.1016/j. ccep.2018.11.001

8. Price S, Platz E, Cullen L, et al. Expert consensus document: echocardiography and lung ultrasonography for the assessment and management of acute heart failure. Nat Rev Cardiol. 2017;14(7):427-440. doi:10.1038/nrcardio. 2017.56

9. Nussbaumerova B, Rosolova H. Diagnosis of heart failure: the new classification of heart failure. Vnitr Lek. 2018;64(9):847-851. doi: $10.36290 / \mathrm{vnl} .2018 .116$

10. Hopkins BD, Hodakoski C, Barrows D, Mense SM, Parsons RE. PTEN function: the long and the short of it. Trends Biochem Sci. 2014;39(4):183-190. doi:10.1016/j.tibs.2014.02.006

11. Chen CY, Chen J, He L, Stiles BL. PTEN: tumor suppressor and metabolic regulator. Front Endocrinol. 2018;9:338. doi:10.3389/ fendo.2018.00338
12. Alvarez-Garcia V, Tawil Y, Wise HM, Leslie NR. Mechanisms of PTEN loss in cancer: it's all about diversity. Semin Cancer Biol. 2019;59:66-79. doi:10.1016/j.semcancer.2019.02.001

13. Agbo E, Liu D, Li M, et al. Modulation of PTEN by hexarelin attenuates coronary artery ligation-induced heart failure in rats. Turk J Med Sci. 2019;49(3):945-958. doi:10.3906/sag-1812-49

14. Yd W, Tt W. Clinical efficacy of trimetazidine hydrochloride combined with metoprolol in the treatment of ischemic cardiomyopathy with heart failure. Chin J Gerontol. 2018;38(6):1333-1335.

15. Livak KJ, Schmittgen TD. Analysis of relative gene expression data using real-time quantitative PCR and the 2(-Delta Delta C(T)) method. Methods. 2001;25(4):402-408. doi:10.1006/meth.2001.1262

16. Gu LP, Hu J, Yan SH. Efficacy of metoprolol and trimetazidine in the treatment of heart failure in elderly patients with coronary heart disease and their effects on cardiac function, myocardial remodeling and inflammatory factors. Chin J Gerontol. 2017;37(1):89-91.

17. Liu S, Jia J, Zhou H, et al. PTEN modulates neurites outgrowth and neuron apoptosis involving the PI3K/Akt/mTOR signaling pathway. Mol Med Rep. 2019;20(5):4059-4066. doi:10.3892/mmr.2019.10670

18. Hu H, Wu J, Li D, Zhou J, Yu H, Ma L. Knockdown of IncRNA MALAT1 attenuates acute myocardial infarction through miR-320PTEN axis. Biomed Pharmacother. 2018;106:738-746. doi:10.1016/ j.biopha.2018.06.122

19. Papadimitriou L, Georgiopoulou VV, Kort S, Butler J, Kalogeropoulos AP. Echocardiography in acute heart failure: current perspectives. $J$ Card Fail. 2016;22(1):82-94. doi:10.1016/j. cardfail.2015.08.001

20. Pastore MC, Mandoli GE, Aboumarie HS, et al. Basic and advanced echocardiography in advanced heart failure: an overview. Heart Fail Rev. 2020;25(6):937-948. doi:10.1007/s10741-019-09865-3

21. Suo LM, Si NN, Jin L, et al. [Evaluation of curative effect of VNG and VEMP in patients with severe sudden hearing loss]. Lin Chung Er Bi Yan Hou Tou Jing Wai Ke Za Zhi. 2018;32(14):1102-1105. Chinese. doi:10.13201/j.issn.1001-1781.2018.14.015

22. Ruddox V, Sandven I, Munkhaugen J, Skattebu J, Edvardsen T, Otterstad JE. Atrial fibrillation and the risk for myocardial infarction, all-cause mortality and heart failure: a systematic review and meta-analysis. Eur J Prev Cardiol. 2017;24(14):1555-1566. doi:10.1177/2047487317715769

23. Bahit MC, Kochar A, Granger CB. Post-myocardial infarction heart failure. JACC Heart Fail. 2018;6(3):179-186. doi:10.1016/j. jchf.2017.09.015

24. Yang H, Wang XX, Zhou CY, et al. Tripartite motif 10 regulates cardiac hypertrophy by targeting the PTEN/AKT pathway. $J$ Cell Mol Med. 2020;24(11):6233-6241. doi:10.1111/jcmm.15257

25. Nodari S, Metra M, Dei Cas L. Beta-blocker treatment of patients with diastolic heart failure and arterial hypertension. A prospective, randomized, comparison of the long-term effects of atenolol vs. nebivolol. Eur J Heart Fail. 2003;5(5):621-627. doi:10.1016/ S1388-9842(03)00054-0
International Journal of General Medicine

\section{Publish your work in this journal}

The International Journal of General Medicine is an international, peer-reviewed open-access journal that focuses on general and internal medicine, pathogenesis, epidemiology, diagnosis, monitoring and treatment protocols. The journal is characterized by the rapid reporting of reviews, original research and clinical studies across all disease areas. The manuscript management system is completely online and includes a very quick and fair peer-review system, which is all easy to use. Visit http://www.dovepress.com/ testimonials.php to read real quotes from published authors. 